\begin{tabular}{|c|c|}
\hline Citation & $\begin{array}{l}\text { Xin Wang, Julian Stoev, Gregory Pinte, Jan Swevers (2013) } \\
\text { Classical and Modern Methods for Time-constrained Energy } \\
\text { Optimal Motion - } \\
\text { Application to a Badminton Robot } \\
\text { Mechatronics, Volume 23, Issue 6, Pages 669-676. }\end{array}$ \\
\hline Archived version & $\begin{array}{l}\text { Author manuscript: the content is identical to the content of the } \\
\text { published paper, but without the final typesetting by the publisher }\end{array}$ \\
\hline Published version & http://dx.doi.or/10.1016/j.mechatronics.2013.07.001 \\
\hline Journal homepage & \\
\hline Author contact & $\begin{array}{l}\text { xin.wang@mech.kuleuven.be } \\
\text { phone number + } 32(0) 16322222\end{array}$ \\
\hline IR & https://lirias.kuleuen.be/handle/123456789/404835 \\
\hline
\end{tabular}




\title{
Classical and Modern Methods for Time-constrained Energy Optimal Motion - Application to a Badminton Robot
}

\author{
Xin Wang ${ }^{1}$ and Jan Swevers \\ Department of Mechanical Engineering, KU Leuven, Mailbox 2420 Celestijnenlaan 300B, B-3001 Heverlee, Belgium \\ Email: xin.wang@mech.kuleuven.be,Jan.Swevers@mech.kuleuven.be \\ Tel: +32(0)1632 2833, Fax: +32(0)16322987 \\ Julian Stoev and Gregory Pinte \\ Flanders' Mechatronics Technology Centre, Celestijnenlaan 300 D, 3001 Leuven, Belgium \\ Email: julian.stoev@fmtc.be, gregory.pinte@fmtc.be
}

\begin{abstract}
Two control approaches are presented to improve the energy efficiency of a robot which has to perform point-to-point motions during a fixed time interval. One is called Proximate Energy Optimal Servo (PEOS) and the other is called Energy-Optimal Model Predictive Control (EOMPC). The PEOS approach is based on the Proximate Time Optimal Servo (PTOS) control algorithm whose parameters are optimized in order to achieve energy efficient behavior. The EOMPC approach developed based on the Time-Optimal Model Predictive Control (TOMPC) approach achieves energy optimal performance by minimizing the energy losses in the object function. The two developed approaches are applied to a robot playing badminton and compared to two existing control approaches: the Proximate Time Optimal Servo (PTOS) and the Time-Optimal Model Predictive Control (TOMPC). The robot is still able to intercept most of the opponent shuttles on time, while a significant reduction of the energy consumption is demonstrated in both cases.
\end{abstract}

Keywords: Motion control, Model based control, Time-optimal control, Energy optimal control, Embedded mechanical systems.

\section{Introduction}

Time-optimal motion control $[1,2,3]$ received large attention both in the academic and the industrial world due to the typical requirement for fast and accurate motions when maximizing the system's productivity. Proximate Time-Optimal Servo (PTOS) control $[4,5]$ is widely used for point-to-point motion control. Recently time-optimal Model Predictive Control (TOMPC) [6] is proposed as an alternative control scheme, where all the system constraints such as actuator saturation and other non-linear effects can explicitly be taken into account. Energy-optimal motion control is also a well researched area with many references like $[7,8,9,10,11]$. Due to the increasing energy prices and the growing social awareness to achieve an environmentally sustainable future, there is a growing industrial interest in energy efficient systems. Since time- and energy-optimality are often conflicting requirements, multi-objective optimiza-

Preprint submitted to Mechatronics tion [12] can be used to find the optimal trade-off between these requirements. Current results indicate that for many industrial machines, energy saving is often possible while preserving the essential productivity of the machine.

In this paper two approaches are presented. The first one is a classical approach, where the settings of an existing PTOS motion controller are adapted for each motion, such that less energy is consumed. This method is further referred to as Proximate Energy Optimal Servo (PEOS). The optimization objective is to achieve energy-optimality while maintaining motion time requirements. The main advantage of this approach is its computational simplicity. The second approach is a modern approach, based on on-line optimization, more precisely Model Predictive Control (MPC). This method is further referred to as Energy $\mathrm{Op}$ timal Model Predictive Control (EOMPC), the basis of 
which is time-optimal MPC (TOMPC) [6]. The computational requirements for this approach are higher, but measurably better performance is obtained. Outline of the paper: The paper starts with a brief description of our experimental plant - a badminton robot with existing PTOS motion controller. The power consumption model related to our robot is specified. Then the modified PEOS approach is described, followed by the description of the EOMPC. Section 4 discusses the experimental validation of both developed approaches and critically evaluates the obtained results. In addition, a comparison of PEOS, PTOS, EOMPC and TOMPC is provided. The conclusions are drawn in the last section.

\section{Experimental setup}

\subsection{Design of the badminton robot}

Fig. 1 shows a schematic representation of the experimental test setup. A detailed description of the robot and its subsystems can be found in [13]. The robot has 3 degrees of freedom - a linear axis, a rotational axis and a hit axis. The linear axis is used to position the robot across the field using a linear motor which is the main energy consumer. The energy consumption of the other axes is neglected. For this reason, the time-constrained energy optimal point-to-point motion control of this linear motor is the subject of this paper.

The dynamics of this linear motor relating the motor current and position $y[m]$ are modelled as a double integrator, and hence this model neglects viscous friction which is an approximation. In model Eq. (1), the position $y[m]$ is output, the acceleration $a\left[m / s^{2}\right]$ is input $u$ and $v[\mathrm{~m} / \mathrm{s}]$ is the velocity.

$$
\begin{aligned}
& \dot{y}(t)=v(t) \\
& \dot{v}(t)=a(t)=u(t)
\end{aligned}
$$

In this paper, this model Eq. (1) is considered for the PEOS approach. However, for the EOMPC approach, a discrete-time state-space model is required. For this reason, the model used for EOMPC is the zero-orderhold discrete-time equivalent of model (1), given in Eq. (2). The selected sampling frequency is $100[\mathrm{~Hz}]$.

$$
\begin{aligned}
\mathbf{x}_{k+1} & =A \mathbf{x}_{k}+B u_{k} \\
y_{k} & =C \mathbf{x}_{k}=\mathbf{x}_{k}(2) \\
v_{k} & =C_{v} \mathbf{x}_{k}=\mathbf{x}_{k}(1)
\end{aligned}
$$

in which

$A=\left[\begin{array}{cc}1 & 0 \\ 0.01 & 1\end{array}\right], B=\left[\begin{array}{c}0.01 \\ 0.00005\end{array}\right], C=\left[\begin{array}{ll}0 & 1\end{array}\right], C_{v}=\left[\begin{array}{ll}1 & 0\end{array}\right]$



Figure 1: Concept of the robot playing field and the visual system

and $k$ is the discrete time index. Input $u$ is acceleration $a\left[m / s^{2}\right]$, velocity $v[m / s]$ and position $y[m]$ are the first and the second state variables of the system respectively.

$a, v$ and $y$ are bounded by their corresponding maximum values - maximum acceleration $\hat{a}$, maximum velocity $\hat{v}$ and maximum position $\hat{y}$, imposed by the physical limitations of the system. Hence, the constrains on $a, v$ and $y$ shown in Eq. (3) are taken into account.

$$
-\hat{y},-\hat{v},-\hat{a} \leq y, v, u \leq \hat{y}, \hat{v}, \hat{a}
$$

A stereo camera system is used to detect the shuttle cock. Based on shuttlecock location measurements, the shuttle cock trajectory is estimated. Interception logic determines the appropriate hit time and the robot configuration (i.e. hit angle, rotational angle and position along the linear axis) in order to hit back the shuttle to the human opponent at a point along the predicted shuttlecock trajectory. After the hit, the robot moves back to the homing position, the center of the linear motor, with a given constant homing time and waits for the next desired setpoint requested. Because the shuttlecock trajectory is known with a large uncertainty immediately after the human player has hit the shuttlecock, the interception point (position and time) estimation is repeated continuously during the motion. Since these estimates can change drastically during the motion, the developed control algorithms must be able to deal with continuously changing interception points.

\subsection{The robot power consumption}

A power consumption model for the linear axis of the robot has been established experimentally. First, energy measurements were performed on the 3-phase entrance of the electrical cabinet of the robot. During an approximately 500 seconds, the three currents and voltages and 
robot position along the linear axis were measured while the linear motor performs a sequence of typical pointto-point motions. Energy consumption estimates are hence obtained by multiplying and adding corresponding phase current and voltage samples. Ethercat devices were used for high sampling rate analog-digital conversion. These energy consumption estimates were also verified using dedicated high precision, slow sampling energy measurement equipment. By differentiating and double differentiating the synchronously recorded position measurements, estimates of velocity and acceleration were obtained. The resulting set of velocity, acceleration, and energy consumption estimates are hence used to identify an power consumption model for the linear motor. The structure of this model is determined based on the following physical considerations:

- Due to the fact that acceleration is proportional to linear motor forces for the badminton robot (mainly inertial forces), which is on itself proportional to motor currents, and resistive losses are proportional to the square of the current, it is reasonable to assume that power consumption model contains an acceleration squared term.

- Due to the fact that viscous friction losses are related to the velocity of the linear motor and corresponding power consumption are equal to force times velocity, an velocity squared term is added to the power consumption model of the motor.

- Due to the presence of Coulomb friction, which depends on the sign of the velocity, also the absolute value of the velocity is part of the power consumption model.

Based on this physical reasoning, first a regression model depending on all possible monomials of velocity $v$, acceleration $a$, and $|v|$ up to degree 2 (for example $a^{2}$, $\left.a \times v, v^{2}, a, v, \cdots\right)$ was explored, and the statistical contribution of each factor was evaluated. This statistical analysis revealed that losses due to Coulomb friction are negligible for this system, and only the following terms significantly contribute to the power consumption: $v^{2}$, $a^{2}$, and $a \times v$. The first two term are motivated above. The term $a \times v$ relates to the mechanical power that is put in the robot and is being transformed into (or from) kinetic energy of the moving mass. Eq. (4) shows the selected power consumption model structure:

$$
P=c_{0}+c_{1} \times v^{2}+c_{2} \times a \times v+c_{3} \times a^{2}
$$

The energy consumption is obtained by integration of Eq. (4) over time. The parameters $c_{i}(i=0, \cdots, 3)$ of this model are estimated in a linear least squares sense using the experimental data discussed below. Table 1 presents the estimated parameters. Figure 2 shows

Table 1: Estimated parameters

\begin{tabular}{|c|c|c|c|}
\hline $\begin{array}{c}c_{0} \\
{[W]}\end{array}$ & $\begin{array}{c}c_{1} \\
{\left[W s^{2} / m^{2}\right]}\end{array}$ & $\begin{array}{c}c_{2} \\
{\left[W s^{3} / m^{2}\right]}\end{array}$ & $\begin{array}{c}c_{3} \\
{\left[W s^{4} / m^{2}\right]}\end{array}$ \\
\hline 0.120 & 0.081 & 0.062 & 0.005 \\
\hline
\end{tabular}

the experimental validation of the identified power consumption model (4) for a set of point-to-point motions executed using the PTOS [5] controller and the PEOS controller (discussed below). Using PTOS and PEOS, the model-based energy predictions are $247.7[\mathrm{~kJ}]$ and $115.1[k J]$ respectively and the energy consumption estimates based on 3-phase current and voltage measurement are $247.0[k J]$ and $115.7[k J]$ respectively. Hence, in this case, the prediction errors are less than $1.0 \%$ which is small. The model-based energy consumption predictions and the energy consumption estimates based on 3-phase current and voltage measurement match quite well for both controllers.

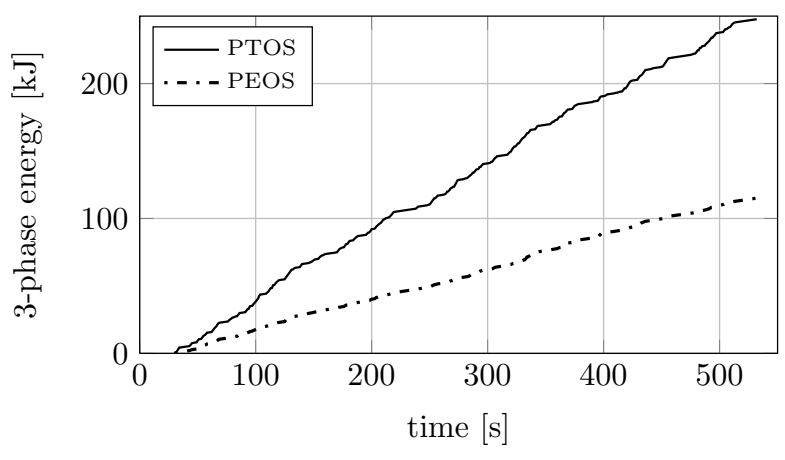

Figure 2: Model-based energy consumption predictions

\section{Energy optimal motion control}

Fig. 3 shows the general control scheme of the badminton robot. A trajectory and feedforward generator provides the trajectory $y_{r}$ and the feedforward signal $u_{f f}$ to the robot. A position feedback tracking controller is utilized to guarantee stable and accurate positioning. The sampling rate of the feedback control loop is $1 \mathrm{kHz}$.

Both the PEOS and the EOMPC approaches discussed below are used as the trajectory and feedforward 


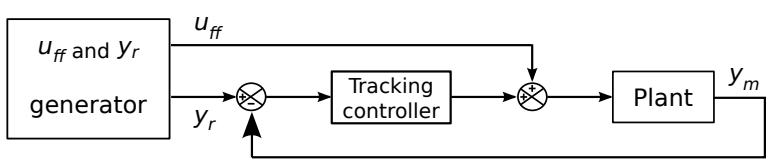

Figure 3: Control structure of the badminton robot linear axis

generator in this configuration, i.e. are used in combination with the tracking controller in order to perform energy-optimal point-to-point motions.

\subsection{PEOS with Genetic Algorithm (GA) tuning}

Since PTOS [5] is the basis of our PEOS approach, this algorithm is first presented. The PTOS algorithm generates control signals $u(t)$ for time-optimal point-topoint motions assuming ideal double integrator system dynamics (1) and for given constraints $\hat{v}$ and $\hat{a}$ on the velocity and acceleration respectively. If the goal is to move from $y=0$ to a desired position $y=y_{\text {des }}$, the PTOS control signal $u$ has the following form:

$$
\begin{aligned}
& u(t)=\hat{a s a t}_{1}\left(k_{2}\left(\operatorname{sat}_{\hat{v}}\left(f_{\text {ptos }}(e(t))\right)-v(t)\right)\right) \\
& e(t)=y_{d e s}-y(t)
\end{aligned}
$$

where

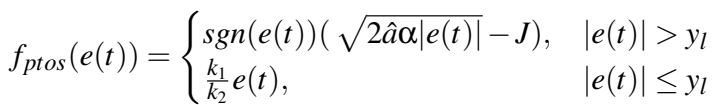

$$
\begin{aligned}
& k_{1}=\frac{2 \alpha \xi^{2}}{y_{l}}, k_{2}=2 \xi^{2} \sqrt{\frac{2 \alpha}{\hat{a} y_{l}}}, J=\frac{1}{\sqrt{\frac{2}{\hat{a} \alpha y_{l}}}} \\
& \operatorname{sat}_{X}(x)=\left\{\begin{array}{lc}
-x, & x<-x \\
x, & x>x \\
x, & \text { otherwise }
\end{array}\right.
\end{aligned}
$$

The controller parameters $k_{1}, k_{2}, J$ depend on the design parameters $y_{l}, \xi$, $\alpha$ which can be used to gain robustness at the cost of a small loss of time optimality. $y_{l}$ is the size of the region near the target $y_{d e s}$, where the PTOS algorithm behaves like a linear controller. In the linear region $\xi$ is the desired damping of the closed loop system. $\alpha \leq 1$ is a discount factor to obtain a safety margin during the deceleration.

The total time available for the badminton motion is limited by the expected fly-time of the badminton shuttlecock (usually less than $1 \mathrm{sec}$ ). For this reason originally a PTOS algorithm was implemented for the trajectory and feed-forward generation of the linear axis of the robot, as shown on Fig. 2. This algorithm aims to

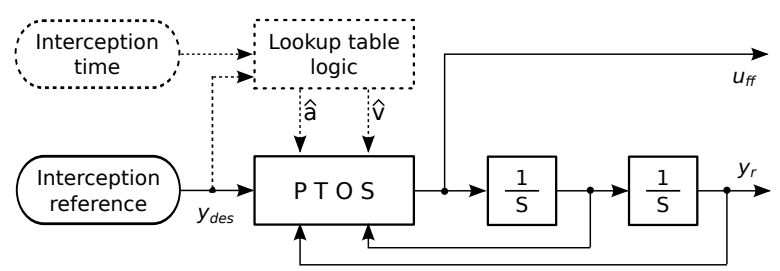

Figure 4: PTOS (solid line) and PEOS (solid and dashed line) used as trajectory and feedforward generator

position this axis in the hit configuration as fast as possible taking into account actuator constraints. As a result the robot arrives in most cases too early and consumes high amounts of energy.

According to (4) an effective method to reduce energy losses, is to reduce the velocity and acceleration of the linear axis during the motion of the robot. The idea in the PEOS approach is to adapt the $\hat{v}$ and $\hat{a}$ parameters in the PTOS equation (5) for each hit of the robot depending on the available time and the distance to be traveled in order to increase the energy efficiency. While in the original PTOS scheme $\hat{a}$ and $\hat{v}$ are constant and determined by the physical limitations of the system, in the new scheme in Fig. 4 the interception point and time are processed by lookup tables in order to modify $\hat{a}$ and $\hat{v}$. A practical method for the off-line calculation of these lookup tables is presented in the following steps.

1. Calculation of optimal values for $\hat{v}$ and $\hat{a}$ using a multi-objective Genetic Algorithm (GA) for a large set of motion times and distances.

2. Construction of lookup tables from the large set of optimized $\hat{v}$ and $\hat{a}$ values through spatial averaging.

It is important to note that the fundamental structure of PTOS and PEOS are the same and hence no significant increase of the online computational complexity is introduced by the proposed modification.

\subsubsection{Multi-objective tuning of $\hat{v}$ and $\hat{a}$ in simulation}

The core of our approach is the formulation of the selection of optimal values for $\hat{a}$ and $\hat{v}$ as a multi-objective optimization problem, trading-off the energy $E$ consumed during a point-to-point motion, the corresponding motion time $T$ and at the same time the travelled distance $y_{d e s}$ that has to be maximized. We note this multi-objective problem as $\left(E \downarrow, T \downarrow, y_{d e s} \uparrow\right)$. A practical and relatively simple approach to solve such problems is using a Multi-Objective Genetic Algorithm (MOGA) $[14,15]$. 
For this purpose each individual is parametrized by a chromosome containing a triplet $\left(\hat{a}, \hat{v}, y_{\text {des }}\right)$. The cost function used to minimize the values in the sense of Pareto has the following form

$$
\left(\hat{a}, \hat{v}, y_{\text {des }}\right) \stackrel{\text { cost }}{\longrightarrow}\left(E, T, \frac{1}{y_{\text {des }}}\right),
$$

where the problem $\left(E \downarrow, T \downarrow, y_{\text {des }} \uparrow\right)$ is replaced with the equivalent $\left(E \downarrow, T \downarrow, 1 / y_{\text {des }} \downarrow\right)$ problem. $E$ and $T$ are calculated using motion data obtained by simulating the linear motor axis model (1), in closed loop configuration Fig. 3 with PTOS as reference and feedforward generator for given values of $y_{d e s}, \hat{v}$ and $\hat{a}$. The motion time $T$ directly follows from the simulation, the consumed energy $E$ is calculated using equation (4) with the values from Table 1. An abstract representation of the function that calculates $T$ and $E$ is given by equation (8):

$$
\left(\hat{a}, \hat{v}, y_{\text {des }}\right) \stackrel{\text { simulation }}{\longrightarrow}(E, T)
$$

The described multi-objective genetic algorithm was run for 50 generations, each of 200 individuals with the cost function (7). The result is a 3-d Pareto front in $\left(E, T, y_{\text {des }}\right)$ space represented by a large number of optimal solutions of the multi-objective problem $(E \downarrow, T \downarrow$ ,$\left.y_{\text {des }} \uparrow\right)$ like in $[14,15]$.

\subsubsection{Constructing the $\hat{a}$ and $\hat{v}$ lookup tables}

For each point $\left(E, T, y_{d e s}\right)$ on the above Pareto front, the corresponding acceleration and velocity values from the chromosome triplet $\left(\hat{a}, \hat{v}, y_{\text {des }}\right)$ are available. This permits us to construct the functional maps

$$
\begin{aligned}
& \left(T, y_{\text {des }}\right) \quad \mapsto \hat{a} \\
& \left(T, y_{\text {des }}\right) \mapsto \hat{v}
\end{aligned}
$$

which can be used to determine the PTOS settings ( $\hat{a}$, $\hat{v})$. Alternatively $\mathrm{E}$ and $\mathrm{T}$ can be obtained from measurements on the real robot. To construct these maps, the points from the Pareto front above were processed as follows. The procedure performs spatial averaging of the Pareto front points, where the goal is to obtain robust low dimensional lookup tables representing sufficiently well the functional maps. The spatial averaging procedure consists the following steps:

1. The $\left(T, y_{\text {des }}\right)$-plane is divided into a grid, consisting of all combinations from the sets $T \in\{0.75,1,1.25,1.5\}, \quad y_{\text {des }} \in$ $\{0.4,0.6,0.8,1,1.25,1.5,1.75,2,2.25\}$.

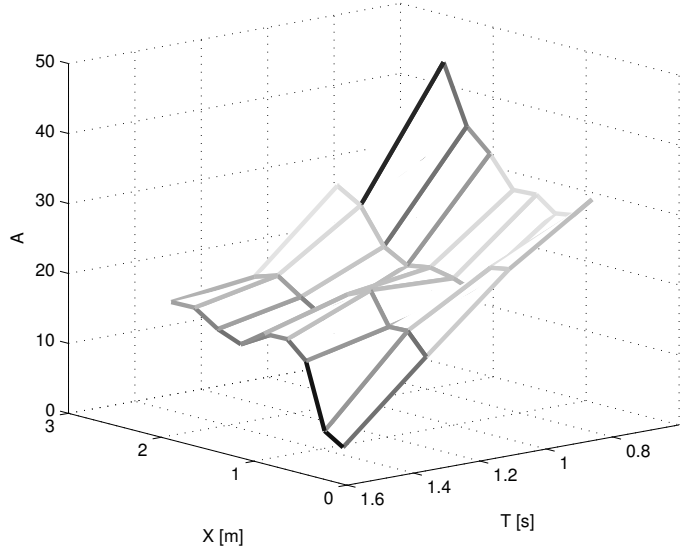

Figure 5: Maximum acceleration lookup table $\left(T, y_{\text {des }}\right) \mapsto \hat{a}$

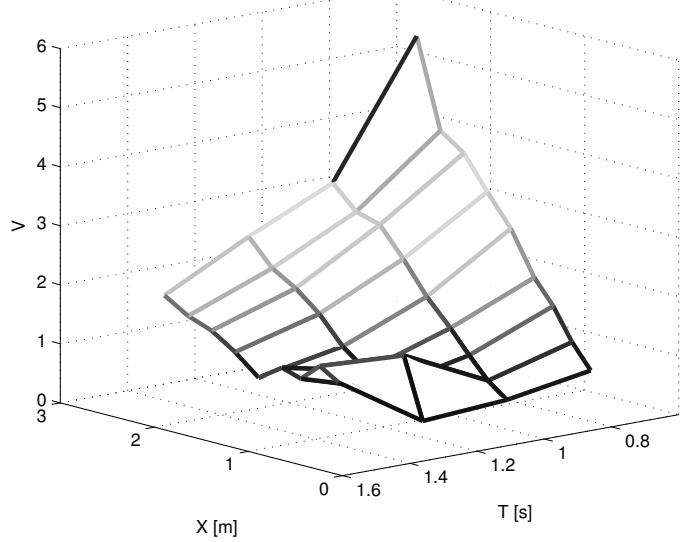

Figure 6: Maximum velocity lookup table $\left(T, y_{\text {des }}\right) \mapsto \hat{v}$

2. For each point of the grid, the 50 closest points from the Pareto front are selected using Euclidean metric.

3. Two linear surface fits are performed of these 50 points - one for the $\left(T, y_{d e s}\right) \mapsto \hat{a}$ relationship and another for $\left(T, y_{\text {des }}\right) \mapsto \hat{v}$, resulting in two sets of linear approximating coefficients. The linear surfaces values are then re-evaluated on the grid point $\left(T, y_{d e s}\right)$ and the resulting are stored in the lookup table cell corresponding to the grid point.

The above procedure it performed only once off-line. The resulting $\left(T, y_{\text {des }}\right) \mapsto \hat{a}$ and $\left(T, y_{\text {des }}\right) \mapsto \hat{v}$ of lookup tables are easy and quick to evaluate in real time. They are shown on Fig. 5 and Fig. 6.

\subsection{Energy-Optimal MPC}

The basic concept of the proposed Energy-Optimal MPC (EOMPC) method is similar as in [16]. However, 
compared with [16], some improvements and extensions of the EOMPC method are presented in this paper: (i) a more accurate, experimentally verified power consumption model is used, (ii) more details of the mathematical formulation of the EOMPC QP problem are provided, (iii) a more extensive experimental validation is presented.

\subsubsection{The basis of EOMPC - TOMPC}

Energy-optimal MPC (EOMPC) is developed based on TOPMC [6] which is a MPC approach for timeoptimal point-to-point motion control of linear timeinvariant (LTI) systems. TOMPC is formulated as a two-layer optimization problem. First the settling time, which is defined as the number of discrete time sampling instances required for the system to be at rest at the desired set point, is minimized so that the time optimality is achieved. Once the minimal settling time is determined, the optimal signal sequence is obtained by solving the MPC optimization problem. Details of the TOMPC approach are discussed in [6].

Similar to PTOS and PEOS, TOMPC and EOMPC are used as a reference trajectory $y_{r}$ and feedforward $u_{f f}$ generator as described in Fig. 7 and Fig. 3 considering ideal double integrator system dynamics (2). The sampling rate of the TOMPC/EOMPC is limited to $100 \mathrm{~Hz}$ due to the computational complexity of the MPC realtime optimization. However, the sampling rate used in the feedback control scheme shown in Fig. 3 is $1 \mathrm{kHz}$. Due to this sampling rate difference, sub-sampling is performed on the interception data and zero-order-hold extrapolation is performed on the TOMPC/EOMPC control signals as indicated in Fig. 7. In order to obtain the smooth reference signal $y_{r}$, the zero-order-hold extrapolation is performed in between the two single integrators. Beside the sub-sampling and the zero-orderhold extrapolation which are utilized only in Fig. 7, trajectory and feedforward generator control schemes shown in Fig. 4 and Fig. 7 are exactly the same.

Remark that in case TOMPC is used, the interception time provided by the shuttle cock interception logic is not used because TOMPC always tries to bring the robot to the desired position (interception reference) as soon as possible, regardless of the interception time.

\subsubsection{Two-layer optimization problem}

EOMPC is a control method to realize energyoptimal point-to-point motions within a given motion time. It is essentially using the TOMPC approach described in [6] to guarantee the settling time of the system is equal to the requested interception time while

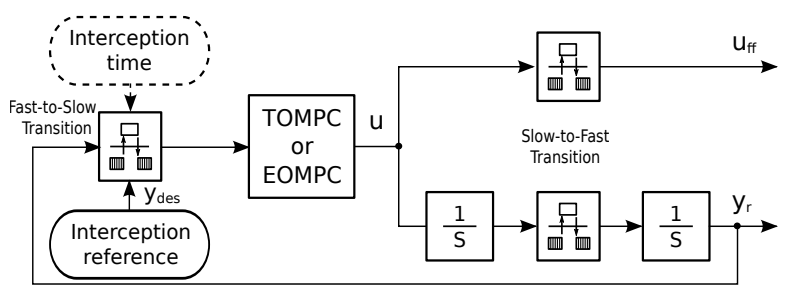

Figure 7: TOMPC (solid line) and EOMPC (solid and dashed line) used as trajectory and feedforward generator

achieving the energy optimality. In the EOMPC the motion time minimization using TOMPC is stopped once the requested interception time is reached. Energy optimality is achieved by setting the object function of the MPC optimization problem equal to the system's energy consumption. As a result, the motion will be executed within the interception time, and not faster, and the energy consumption will be minimized. If for some reason the interception time is too short for the considered system and displacement considering the system constraints, EOMPC approach will perform exactly the same as TOMPC - in this case it will automatically find the shortest possible motion time (which obviously will be larger than the interception time) and realize the motion in a time-optimal way. Because of the real-time solution strategy, the requirements on interception time and interception reference can be changed during the motion.

Similar to the TOMPC, the EOMPC optimization problem is formulated as a two-layer optimization problem. The top layer is called 'Problem B' and determines the settling time. The settling time is defined as the number $N$ of discrete time sampling instances required for the system to be at rest at the desired interception point. Finding the settling time involves solving a series of feasibility problems. This feasibility problem is the second layer and is called 'Problem A'. The optimal control sequence is the last feasible solution of 'Problem A'. The following paragraphs describe 'Problem A' and 'Problem B' in detail.

'Problem $\mathbf{A}^{\prime}$ denoted as $P_{A}\left(\hat{\mathbf{x}}_{l}, N\right)$ is defined in Eq. (10). It calculates the energy optimal control signal for a given $N$ (which is obtained by solving 'Problem B') while respecting the system constraints. 


$$
\begin{gathered}
V_{A}^{*}=\min _{\mathbf{u}} E_{\text {loss }}(\mathbf{u}) \\
\text { s.t. } \quad \mathbf{x}_{0}=\hat{\mathbf{x}}_{l} \\
\mathbf{x}_{k+1}=\mathbf{A}_{k} \mathbf{x}_{k}+\mathbf{B}_{k} u_{k}, \quad k=0,1, \cdots, N_{\max }-1 \\
y_{k}=\mathbf{x}_{k}(2), \quad k=0,1, \cdots, N_{\max } \\
v_{k}=\mathbf{x}_{k}(1), \quad k=0,1, \cdots, N_{\max } \\
-\hat{y},-\hat{v},-\hat{a} \leq y_{k}, v_{k}, u_{k} \leq \hat{y}, \hat{v}, \hat{a} \\
y_{k}=y_{\text {des }}, \quad k=N, \cdots, N+n-1 \\
u_{k}=0, \quad k=N, \cdots, N_{\max }-1
\end{gathered}
$$

In Equation (10), $\mathbf{u}=\left[u_{0}, \ldots, u_{N_{\max }-1}\right]$ is the decision variable over the prediction horizon $N_{\max } . \hat{\mathbf{x}}_{l}$ in Eq. (10b) is the system state at time instance $l$. Since the EOMPC is used here in an open-loop configuration generating reference position $y_{r}$ and acceleration feed forward $u_{f f}, \hat{\mathbf{x}}_{l}$ is obtained through open-loop simulation of the double integrator system model (2). The optimization is based on the discrete-time state-space system model Eq. (10c) with sampling time $T_{s}=0.01[s]$. The two state variables are the position and velocity as shown in Eq. (10d) - (10e). Eq. (10f) specifies the constraints on the position, the velocity, and the acceleration of the system. Eq. $(10 \mathrm{~g})$ - $(10 \mathrm{~h})$ are moving endpoint constraints and impose the system to be at rest at the desired interception point $y_{\text {des }}$ within $N$ time steps.

The discrete-time state-space system dynamic matrices $\mathbf{A}_{k}$ and $\mathbf{B}_{k}$ in Eq. (10c) are time dependent because of the non-equidistant sampling time considered in the prediction horizon, which is called 'Blocking'. Blocking means instead of using equidistant time intervals, non-equidistant time intervals as shown in Eq. (11) are utilized such that a sufficiently large prediction time horizon can be achieved with limited $N_{\max }$.

$$
\underbrace{\left[\begin{array}{lllll}
b_{0} & b_{1} & b_{2} & \cdots & b_{N_{\max }-1}
\end{array}\right]}_{N_{\max }} \times T_{S}
$$

where $b_{k}$ with $k \in\left\{0,1, \cdots, N_{\max }-1\right\}$ is an integer and $b_{k} \geq 1$. Obviously if $b_{0}=b_{1}=\cdots=b_{N_{\max }-1}=1$, the sampling intervals are equidistant. The matrices $\mathbf{A}_{k}$ and $\mathbf{B}_{k}$ corresponding to discrete-time sampling interval $t_{k}=b_{k} \times T_{s}$ are:

$$
\begin{aligned}
& \mathbf{A}_{k}=A^{b_{k}}, \quad k=0,1, \cdots, N_{\max }-1 \\
& \mathbf{B}_{k}=\sum_{i=0}^{b_{k}-1} A^{i} B, \quad k=0,1, \cdots, N_{\max }-1
\end{aligned}
$$

with $A$ and $B$ the discrete-time state-space matrices defined in Eq. (2).
The outcome of problem $P_{A}\left(\hat{\mathbf{x}}_{l}, N\right)$ is that it is either feasible or not. Infeasibility of $P_{A}\left(\hat{\mathbf{x}}_{l}, N\right)$ means that the system can not be at rest at the interception point $y_{\text {des }}$ within $N$ time steps while respecting system constraints. Therefore, an admissible set $\mathbf{X}(N)$ is defined:

$$
\mathbf{X}(N)=\left\{\hat{\mathbf{x}}_{l} \mid P_{A}\left(\hat{\mathbf{x}}_{l}, N\right) \text { is feasible }\right\}
$$

$\mathbf{X}(N)$ is the set of system states from which the interception point can be reached within $N$ time steps, while respecting all system constraints Eq. (10f).

'Problem B' denoted as $P_{B}\left(\hat{\mathbf{x}}_{l}, N\right)$, calculating the settling time $N$, is defined as follows:

$$
\begin{aligned}
V_{B}^{*}\left(\hat{\mathbf{x}}_{l}, K^{*}\right) & =\min N \\
\text { s.t. } \hat{\mathbf{x}}_{l} & \in \mathbf{X}(N) \\
\max \left(N_{\min }, K^{*}\right) & \leq N \leq N_{\max }
\end{aligned}
$$

where $K^{*}$, the index of the sampling interval within the prediction horizon that contains the interception time $T$, is defined as follows:

$$
\begin{gathered}
K^{*}=k, \quad \text { s.t. } \\
\sum_{i=0}^{k} b_{i} \times T_{s}<T, \quad \sum_{i=0}^{k+1} b_{i} \times T_{s} \geq T \\
k \in\left\{0,1, \cdots, N_{\max }-2\right\}
\end{gathered}
$$

In Equation (14), $N$ is bounded by $N_{\max }$ and the maximum of $N_{\min }$ and $K^{*}$. To guarantee unconstrained solvability, $N_{\min }$ should be selected bigger than $n / n_{u}$ with $n$ the number of states and $n_{u}$ the number of inputs [17]. Hence, at each time instance $l$, 'problem B' minimizes $N$ up to $K^{*}$ if $y_{d e s}$ can be reached in $K^{*}$ time steps $\left(P_{A}\left(\hat{\mathbf{x}}_{l}, K^{*}\right)\right.$ is feasible) except if (i) $y_{\text {des }}$ can be reached in less than or equal to $N_{\text {min }}$ time steps with $K^{*}<N_{\text {min }}$, yielding $N=N_{\min }$ or if (ii) $y_{\text {des }}$ can't be reached in $K^{*}$ time steps, yielding $N>K^{*}$.

\subsubsection{Quadratic Problem ( $Q P)$ formulation}

The object function $E_{\text {loss }}$ in Eq. (10a) is based on the experimentally identified energy model Eq. (4) and is formulated as follows:

$$
\begin{aligned}
E_{\text {loss }}=\mathbf{v}^{\mathrm{T}} \mathbf{R}_{\mathbf{1}} \mathbf{v} & +\mathbf{u}^{\mathrm{T}} \mathbf{R}_{\mathbf{2}} \mathbf{v}+\mathbf{u}^{\mathrm{T}} \mathbf{R}_{\mathbf{3}} \mathbf{u} \\
\mathbf{R}_{\mathbf{1}} & =c_{1} * \mathbf{I}_{\mathbf{r}} \\
\mathbf{R}_{\mathbf{2}} & =c_{2} * \mathbf{I}_{\mathbf{r}} \\
\mathbf{R}_{\mathbf{3}} & =c_{3} * \mathbf{I}_{\mathbf{r}}
\end{aligned}
$$

with $c_{1}, c_{2}, c_{3}$, the identified coefficients in Table 1 . Due to the fact that a constant in an object function 
doesn't contribute to the optimal solution, $c_{0}$ is ignored in Eq. (16).

$\mathbf{I}_{\mathbf{r}} \in \mathbf{R}^{N_{\max } * N_{\max }}$ is a diagonal matrix but not an identity matrix due to the blocking. Based on the blocking in Eq. (11), $\mathbf{I}_{\mathbf{r}}$ is defined as follows:

$$
\mathbf{I}_{\mathbf{r}}=T_{s} \times\left[\begin{array}{ccccc}
b_{0} & 0 & 0 & \cdots & 0 \\
0 & b_{1} & 0 & \cdots & 0 \\
0 & 0 & b_{2} & \cdots & 0 \\
\vdots & \vdots & \vdots & \ddots & \vdots \\
0 & 0 & 0 & \cdots & b_{N_{\max }-1}
\end{array}\right]
$$

The $k^{\text {th }}$ element on the diagonal of $\mathbf{I}_{\mathbf{r}}, b_{k-1} \times T_{s}$ is the $k^{\text {th }}$ element in Eq. (11) indicating the duration of the $k^{\text {th }}$ discrete-time interval within the prediction horizon, and is a direct consequence of the fact that model equation (4) represents the power consumption in Watt, and the energy loss $E_{\text {loss }}$ is the integral (summation over time) of this power, expressed in Joule. An advantage of EOMPC is obviously shown here that different models of the energy losses can be easily dealt with by defining different weighting matrices $\mathbf{R}_{\mathbf{1}}, \mathbf{R}_{\mathbf{2}}$ and $\mathbf{R}_{\mathbf{3}}$.

The EOMPC optimization problem is a convex quadratic program (QP). It is solved using the qpOASES [18] open source on-line active set $\mathrm{C}++$ software. In order to use this active set method, the problem is condensed first. Condensing means that all states and related equality constraints are eliminated from the optimization problem such that it depends only on the current state $x_{0}$ and the decision variable $\mathbf{u}$.

As a result of condensing, the velocity $\mathbf{v}$ and the position $\mathbf{y}$ can be written as a function of the input $\mathbf{u}$ as shown in Eq. (18):

$$
\begin{aligned}
\mathbf{v} & =\mathbf{U}_{v} \mathbf{u}+v_{l-1} \\
\mathbf{y} & =\mathbf{Y}_{x} x_{0}+\mathbf{Y}_{u} \mathbf{u}
\end{aligned}
$$

where $v_{l-1}$ is the velocity at the time instance prior to the current time instance, that is at the time instance $l-$ 1. According to the selected sampling intervals shown in Eq. (11), the matrices $\mathbf{U}_{v}, \mathbf{Y}_{x}$ and $\mathbf{Y}_{u}$ are defined as follows:

$$
\mathbf{U}_{v}=T_{s} \times\left[\begin{array}{ccccc}
b_{0} & 0 & 0 & \cdots & 0 \\
b_{0} & b_{1} & 0 & \cdots & 0 \\
b_{0} & b_{1} & b_{2} & \cdots & 0 \\
\vdots & \vdots & \vdots & \ddots & \vdots \\
b_{0} & b_{1} & b_{2} & \cdots & b_{N_{\max }-1}
\end{array}\right]
$$

$$
\begin{aligned}
& \mathbf{Y}_{\mathbf{x}}=\left[\begin{array}{c}
C \\
C A^{b_{0}} \\
C A^{b_{0}+b_{1}} \\
\vdots \\
C A^{b_{0}+\cdots+b_{N_{\max }-1}}
\end{array}\right] \\
& \mathbf{Y}_{u}=\left[\begin{array}{cccc}
0 & 0 & \cdots & 0 \\
b_{0} C A^{s_{0}} B & 0 & \cdots & 0 \\
b_{0} C A^{s_{1}} B & b_{1} C A^{s_{0}} B & \cdots & 0 \\
\vdots & \vdots & \ddots & \vdots \\
b_{0} C A^{S_{N_{\max }-1} B} & b_{1} C A^{S_{N_{\max }-2} B} & \cdots & b_{N_{\max }-1} C A^{s_{0}} B
\end{array}\right]
\end{aligned}
$$

where

$$
s_{k}=\sum_{i=0}^{k} b_{i}-b_{0}, k \in\left\{0,1, \cdots, N_{\max }-1\right\}
$$

The size of this QP is constant and hence independent of the interception reference.

\subsubsection{Optimization procedure}

The algorithmic implementation of 'Problem B' of the EOMPC approach is described in Table 2.

\section{Experimental results}

The two proposed control schemes PEOS and EOMPC were implemented on the badminton robot hardware and tested. Since it is difficult to compare the performance and energy-efficiency of the robot during a real badminton game because the same series of the motions can never be repeated exactly, a standard series of test motions of approximately 10 minutes was recorded and repeated twice on the robot, once using the PEOS and once using the EOMPC approach with $N_{\max }=35$. A total of 84 shuttle hits are performed during the play.

Keeping in mind our original multi-objective formulation, the following criteria are evaluated:

- the energy consumption for a series of the motions

- the on-time arrival of the robot at the interception points

A representative zoom of part of the results are shown in Fig. 8. Remark that the interception time does not necessarily change linearly with time and also the interception reference changes during motion. This is because the interception logic continuously updates the interception point. 
(a)

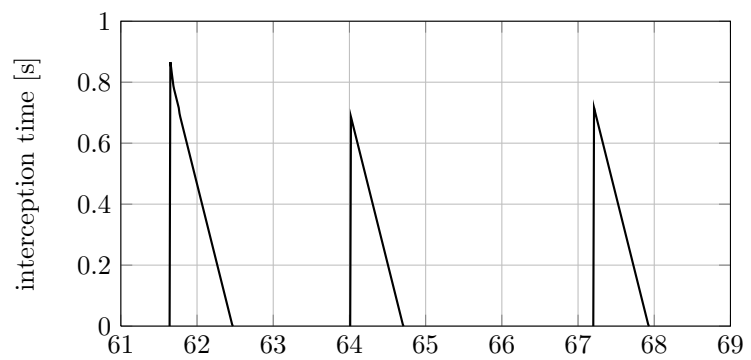

(b)

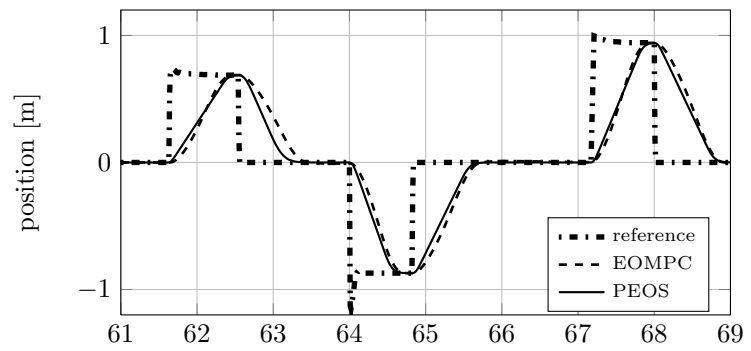

(c)



$(d)$



$(e)$

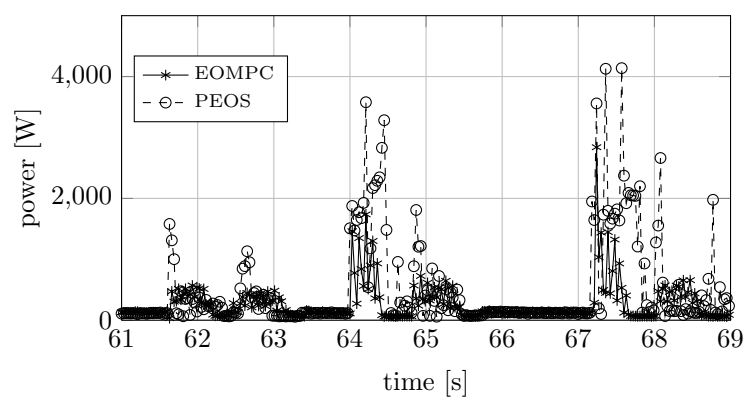

Figure 8: (a) Interception time (b) Motor positions (c) Motor velocity (d) Motor acceleration (e) Motor power
Table 2: Algorithmic implementation of 'Problem B'

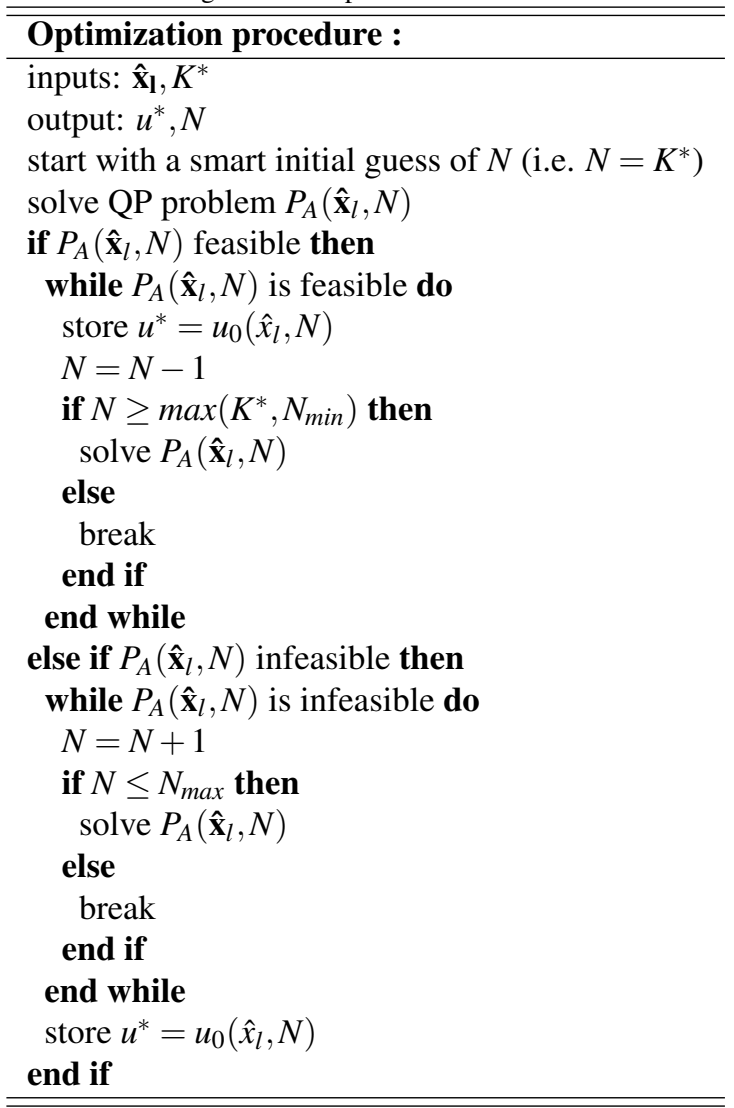

According to the summary of the experimental validation results shown in Table 3, the EOMPC requires less energy than PEOS. For the experiment discussed above, EOMPC and PEOS consume $130 \mathrm{~kJ}$ and $137 \mathrm{~kJ}$ respectively. Fig. 9 shows the positioning error, that is the difference between the actual position at the specified hit time and the interception point, for each of the the 84 requested shuttle hits. Due to the high bandwidth of the position feedback controller, the positioning error is mainly caused by the reference generating (PEOS and EOMPC) algorithms. A positioning error larger than $3 \mathrm{~cm}$ is considered as a missed hit. Fig. 9 clearly shows that the EOMPC, which has 2 missed hits, performs better than the PEOS approach, which has 7 missed hits. This is because that EOMPC is able to trade-off on-time arrival and energy consumption in a more appropriate way than PEOS. EOMPC perfroms an energy optimal motion if the interception time is larger than the possible minimal motion time (corresponding to time-optimal motion), and otherwise automatically performs time optimal motions regardless of the energy consumption. PEOS however is a proximate energy optimal method and it's lookup table is calculated assum- 


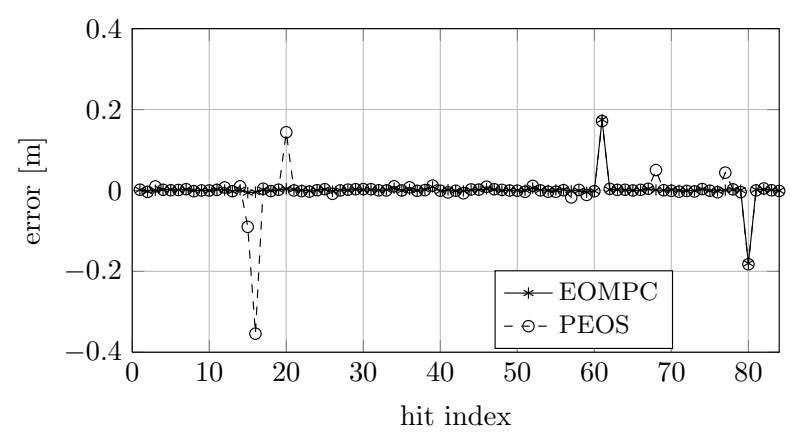

Figure 9: Interception errors at hit moment

ing that initial velocity is equal to zero. This means ontime arrival cannot be ensured using PEOS when initial velocity is nonzero.

Finally, the energy consumption and the hitting performances of the two proposed energy-optimal control scheme and of the PTOS and TOMPC time-optimal control schemes are compared, the summary of which is presented in Table 3. The same zoom part of the results using EOMPC and TOMPC are shown in Fig. 10.

Table 3: Summary of the experimental validation

\begin{tabular}{|c|c|c|}
\hline Method & Missed hits & Measured $E_{\text {loss }}[k J]$ \\
\hline \hline EOMPC & 2 & 130 \\
\hline PEOS & 7 & 137 \\
\hline TOMPC & 2 & 238 \\
\hline PTOS & 2 & 233 \\
\hline
\end{tabular}

TOMPC consumes $238 k J$ with 2 missed hits and PTOS consumes $233 k J$ with 2 missed hits. A huge reduction of the energy, about $40 \%$, is obtained by using EOMPC and PEOS comparing with TOMPC and PTOS. In short, EOMPC achieves the best performance ( 2 missed hits out of 84 ) while consuming the least energy.

\section{Conclusion}

In this paper two approaches for energy optimal motion are presented. The first approach is based on a classical and mature industrial technology, namely PTOS. It is tuned using genetic algorithm with the aim to improve the energy efficiency of the motion. The second approach EOMPC is based on model-predictive control (a)

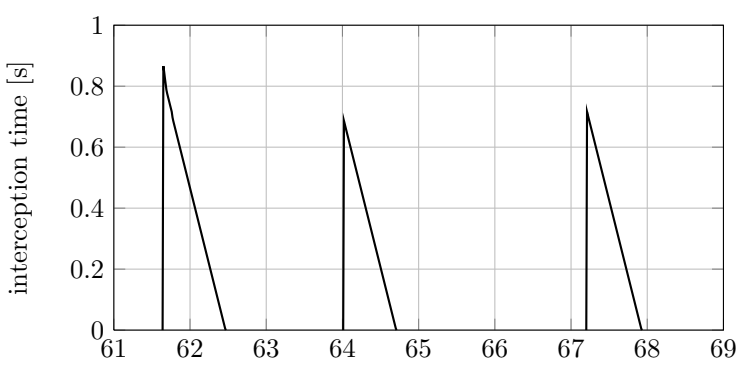

(b)


(d)

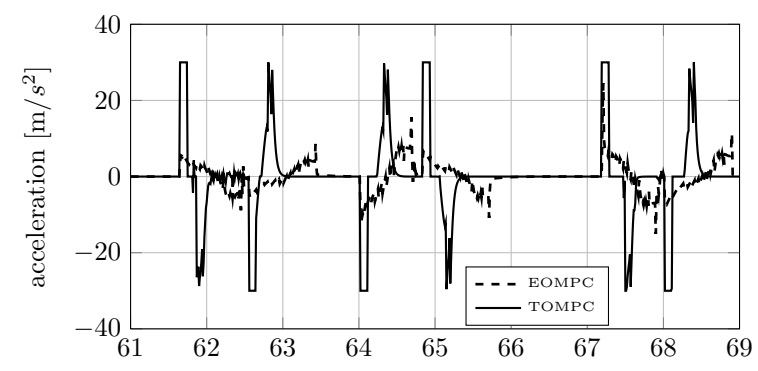

(e)

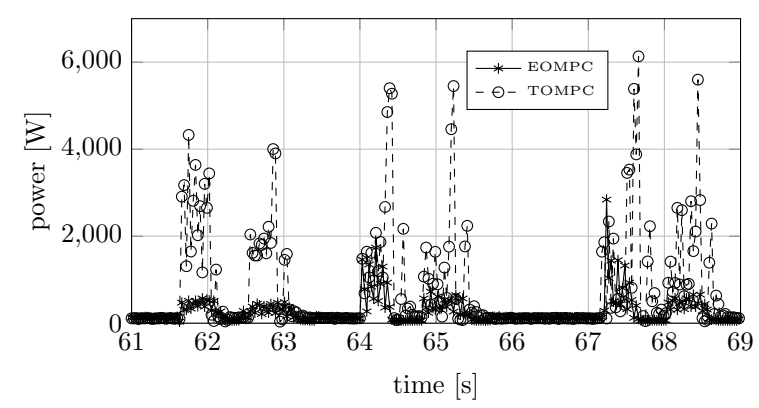

Figure 10: (a) Interception time (b) Motor positions (c) Motor velocity (d) Motor acceleration (e) Motor power 
and includes optimization on each time-step of the cost function based on actual energy of the robot.

Both approaches show very good results. A strong reduction of the dissipated energy is obtained compared to time-optimal control while the performance degradation is negligible. EOMPC outperforms PEOS - both in energy and in precision because the EOMPC can make a good trade-off between the energy consumption and the positioning error. On the other hand, the PEOS approach is much more simple and requires less performant hardware to implement. The EOMPC approach also relies on the availability of cost function describing the energy in a quadratic form, which is probably not possible for all systems.

Future work can apply the approach to higher dimensional systems with more flexible modes. In this case EOMPC, which can deal with high dimension systems, have a very strong advantage with respect to PEOS. Online estimation of the energy cost of the system and corresponding update of the optimization coefficients in the EOMPC could also be another interesting extension.

\section{Acknowledgment}

This work has been carried out within the framework of projects IWT-SBO 80032 (LeCoPro) of the Institute for the Promotion of Innovation through Science and Technology in Flanders (IWT-Vlaanderen). This work also benefits from KU Leuven-BOF PFV/10/002 Center-of-Excellence Optimization in Engineering (OPTEC), the Belgian Programme on Interuniversity Attraction Poles, initiated by the Belgian Federal Science Policy Office (DYSCO), and KU Leuven's Concerted Research Action GOA/10/11 and G.0377.09 of the Research Foundation-Flanders (FWO-Vlaanderen).The research leading to these results has received funding from the European Union Seventh Framework Program (FP7/2007-2013) under grant agreement n247982-ESTOMAD.

\section{References}

[1] Q. Lin, R. Loxton, K. L. Teo, Y. H. Wu, A new computational method for a class of free terminal time optimal control problems, Pacific Journal of Optimization 7 (2011) 63-81.

[2] Q. Lin, R. Loxton, K. L. Teo, Y. H. Wu, Optimal control computation for nonlinear systems with state-dependent stopping criteria, Automatica 48 (2012) 2116-2129.

[3] H. Maurer, N. P. Osmolovskii, Second order sufficient conditions for time-optimal bang- bang control, SIAM Journal on Control and Optimization 42 (2004) 2239-2263.

[4] M. L. Workman, Adaptive proximate time-optimal servomechanisms, Ph.D. thesis, Stanford Univ., Stanford, CA (1987).
[5] A. Dhanda, G. F. Franklin, An improved 2-dof proximate time optimal servomechanism, IEEE Transactions on magnetics 45 (5) (2009) 2151-2164.

[6] L. Van den Broeck, M. Diehl, J. Swevers, A model predictive control approach for time optimal point-to-point motion control, Mechatronics 21(7) (2011) 1203-1212.

[7] B. G. Dijkstra, N. Rambaratsing, C. Scherer, O. H. Bosgra, M. Steinbuch, S. Kerssemakers, Input design for optimal discrete time point-to-point motion of an industrial xy-positioning table, in: Conference on Decision and Control, 2000, pp. 901906.

[8] V. Kluchev, Electric drive theory, Enegroatomizdat, 2001.

[9] E. S. Sergaki, G. S. Stavrakakis, A. D. Pouliezos, Optimal robot speed trajectory by minimization of the actuator motor electromechanical losses, J. Intell. Robotics Syst. 33 (2) (2002) 187207. doi:10.1023/A:1014643401778.

[10] B. Cao, G. Dodds, G. Irwin, Constrained time-efficient and smooth cubic spline trajectory generation for industrial robots, Control Theory and Applications, IEE Proceedings - 144 (5) (1997) 467 -475. doi:10.1049/ip-cta:19971494.

[11] S. F. Woon, V. Rehbock, R. Loxton, Towards global solutions of optimal discrete-valued controlproblems, Optimal Control Applications and Methods 33 (2012) 576-594.

[12] R. Steuer, Multiple criteria optimization: theory, computation, and application, Wiley-Interscience series in systems and optimization, Wiley, 1986.

[13] J. Stoev, A. Bartic, S. Gillijns, W. Symens, Badminton playing robot - a multidisciplinary test case in mechatronics, in: 5th IFAC Symposium on Mechatronic Systems, Marriott Boston Cambridge, USA, 2010. doi:10.3182/20100913-3-US2015.00028 .

[14] N. Srinivas, K. Deb, Multiobjective optimization using nondominated sorting in genetic algorithms, Evolutionary Computation 2 (1994) 221-248.

[15] K. Deb, Multi-objective optimization using evolutionary algorithms, Wiley-Interscience series in systems and optimization, John Wiley \& Sons, 2001.

[16] X. Wang, J. Stoev, G. Pinte, J. Swevers, Energy optimal pointto-point motion using model predictive control, in: 5th annual Dynamic Systems and Control Conference (DSCC), Ft. Lauderdale, FL, USA, 2012.

[17] T. Kailath, Linear systems, Prentice Hall.

[18] H. J. Ferreau, H. G. H.G. Bock, M. Diehl, An online active set strategy to overcome the limitations of explicit MPC, International Journal of Robust and Nonlinear Control 18 (8) (2008) 816-830. 